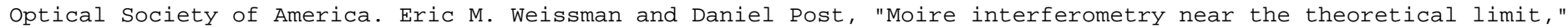
Appl. Opt. 21, 1621-1623 (1982). doi: 10.1364/AO.21.001621

\title{
Moire interferometry near the theoretical limit
}

\author{
Eric M. Weissman and Daniel Post
}

\begin{abstract}
The theoretical upper limit of moire interferometry is approached as the reference grating pitch approaches $\lambda / 2$ and its frequency approaches $2 / \lambda$. This work demonstrates the method at $97.6 \%$ of the theoretical limit. A virtual reference grating of 4000 lines $/ \mathrm{mm}$ (101,600 lines/in.) was used in conjunction with a phase type reflection grating of half of that frequency on the specimen. Sensitivity was $0.25 \mu \mathrm{m} /$ fringe $(9.8 \mu \mathrm{in} . / \mathrm{fringe})$. In-plane displacement fringes of excellent definition were obtained throughout the $76 \times 51$-mm $(3 \times 2$-in.) field of view. They were very closely packed, exhibiting a maximum fringe density of 24 fringes $/ \mathrm{mm}(610$ fringes/in.). Effectiveness of moire interferometry near the theoretical limit was proved.
\end{abstract}

\section{Introduction}

Sensitivity of displacement measurements by moire interferometry increases with the frequency $f$ of the reference grating. A frequency of $f=1200$ lines $/ \mathrm{mm}$ or 30,000 lines/in. was used in Refs. $1-3$ and 1700 lines/mm (43,000 lines/in.) in Ref. 4. Real ${ }^{2,3}$ and virtual $^{1,4}$ reference gratings were employed. While moire interferometry has been applied very successfully with the above cited reference grating frequencies, an upper limit has not been established. The purpose of this work is to demonstrate that moire interferometry is effective even as it approaches its theoretical upper limit.

Figure 1 illustrates a convenient optical configuration for moire interferometry; other equivalent arrangements may be used. The specimen is illuminated symmetrically with two mutually coherent collimated beams at angles $\alpha$ and $-\alpha$, where

$$
\sin \alpha=\frac{\lambda}{2}(f),
$$

and where $\lambda$ is the wavelength of light employed. Intersection of the two beams generates a virtual reference grating comprised of walls of constructive and destructive interference ${ }^{5}$ where the distance between adjacent walls is $g$, and the frequency of the virtual grating is $f=1 / g$.

The authors are with Virginia Polytechnic Institute \& State University, Department of Engineering Science \& Mechanics, Blacksburg, Virginia 24061.

Received 23 November 1981.

0003-6935/82/091621-03\$01.00/0.

(c) 1982 Optical Society of America.
The specimen is prepared with a diffraction grating of frequency $f / 2$ by a relatively easy replication process. ${ }^{1,6}$ The combination of a virtual reference grating of frequency $f$ and a specimen grating of frequency $f / 2$ is a case of moire fringe multiplication ${ }^{5}$ by a factor of 2 . The local frequency and orientation of the specimen grating changes from point to point when the specimen is loaded or otherwise deformed, but the virtual reference grating remains unchanged.

Two beams diffracted by the specimen grating in its +1 and -1 diffraction orders emerge essentially normal to the grating and enter the camera. They combine in two-beam interference to generate the moire interferometry pattern of specimen displacements. Fringe order $N$ at a point in the moire interferometry pattern is proportional to the displacement $U$ of the same specimen point. Specifically,

$$
U=N g=N / f,
$$

where $U$ is the component of in-plane specimen displacement in a direction perpendicular to the lines (or walls) of the reference grating, and $f$ is the frequency of the reference grating.

\section{Theoretical Limit}

Equation (1) prescribes that the upper limit of reference grating frequency is approached as angle $\alpha$ approaches $90^{\circ}$. In the limit its frequency is $f=2 / \lambda$ and its pitch is $g=\lambda / 2$.

In the experiment described below angle $\alpha$ was $77.4^{\circ}$. Since $\sin 77.4^{\circ}=0.976$, the frequency and corresponding sensitivity was $97.6 \%$ of the theoretical limit.

The Ar laser line of wavelength $\lambda=488 \mathrm{~nm}$ was used, not because high power was required but because a low visible wavelength was desired. With this combination of $\alpha$ and $\lambda$, frequency of the virtual reference grating was $f=4000$ lines $/ \mathrm{mm}=101,600$ lines/in. 


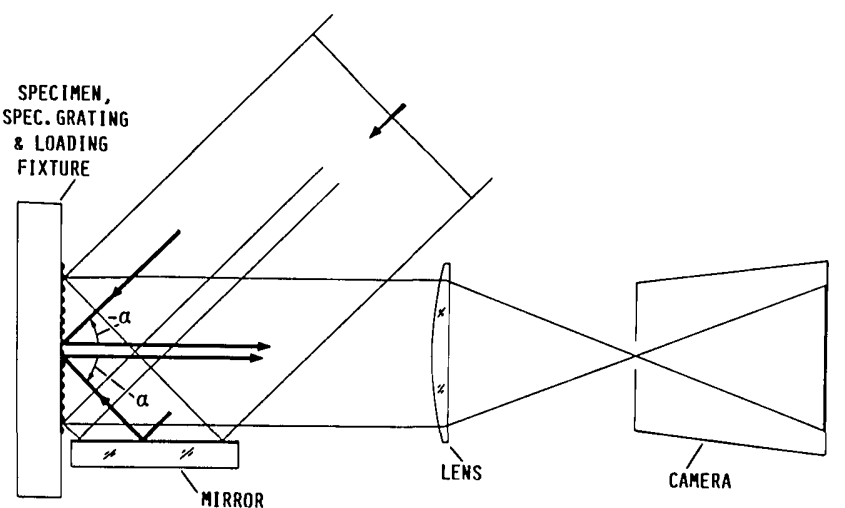

Fig. 1. Optical arrangement for moire interferometry.

\section{Specimen and Specimen Grating}

The specimen was a tensile plate of polymethyl methacrylate $200 \times 51 \times 3.2 \mathrm{~mm}(8 \times 2 \times 0.125 \mathrm{in}$.), with a 10-mm (0.40-in.) diam central hole. A phase type reflection grating of 2000 lines/mm (50,800 lines/in.) was applied to the $76-\times 51-\mathrm{mm}(3-\times 2$-in.) central area illustrated in Fig. 2.

The grating was formed on the specimen by a replication method using a specially prepared master grating as a mold. ${ }^{6}$ The master grating was a photographic plate (Kodak HRP type TE) exposed at the intersection of two collimated beams of coherent light; frequency of the resultant grating is given by Eq. (1). After the plate was developed, bleached, and dried, the unexposed zones (destructive interference zones) exhibited less shrinkage than the exposed zones, causing a lenticulated or corrugated surface of the photographic emulsion with ridges and troughs of predetermined pitch and frequency. The master grating was then coated with a semitransparent film of evaporated aluminum.

For replication an acrylic adhesive (NOL-61, Norland Products Inc., New Brunswick, N.J.) was poured as a small puddle on the specimen, and the master grating was pressed against it to squeeze the adhesive into a thin film of $\sim 0.02-\mathrm{mm}(0.001$-in.) thickness. The adhesive was polymerized by irradiation with ultraviolet light from the master grating side of the assembly. After polymerization only gentle prying was necessary to separate the master grating from the specimen. The weakest interface, however, was between the photographic emulsion surface and the evaporated aluminum, so the aluminum coating adhered to the acrylic cement and was thus transferred to the specimen. ${ }^{6}$ The resulting specimen grating was a furrowed surface with a thin aluminum coating-it was a phase type reflection grating on the specimen.

\section{Experiment and Results}

Figure 1 depicts the experimental arrangement, except that angle $\alpha$ was much larger, namely, $77.6^{\circ}$. The incident collimated beam was formed by an Ar laser (emitting its 488-nm wavelength), a spatial filter, and a parabolic mirror. The specimen was installed in a loading fixture and positioned as shown; the camera was adjusted for 1:1 magnification and for a focused image of the specimen on the camera screen; and the system was aligned ${ }^{1}$ to produce a sparse pattern of moire interferometry fringes. Tensile loads were applied to the specimen, and the pattern was photographed on Kodak Contrast Process Ortho film.

The moire interferometry fringes over the entire 76$\times 51-\mathrm{mm}$ area were well-resolved on the film, but they could not be seen with the naked eye. Magnified views are shown in Fig. 3 for the two areas defined by dashed lines in Fig. 2.

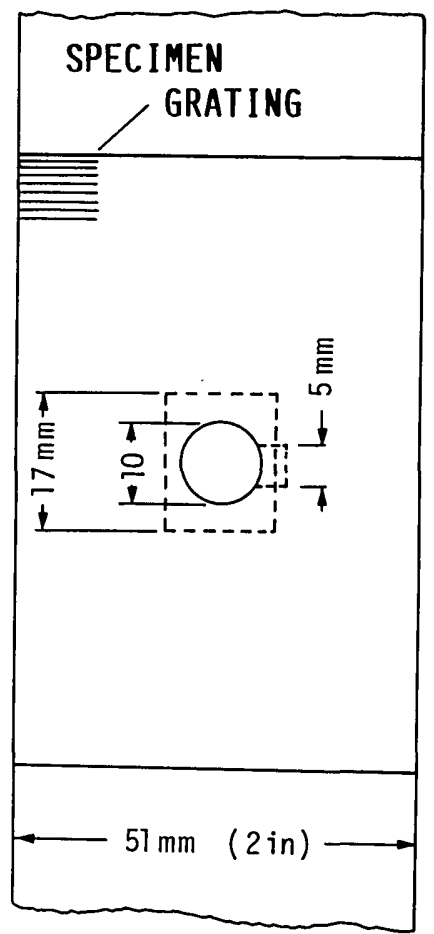

Fig. 2. Tensile specimen $200 \mathrm{~mm}$ (8 in.) long with a central hole. Grating area is $76 \times 51 \mathrm{~mm}$; dashed areas show locations of patterns of Fig. 3.
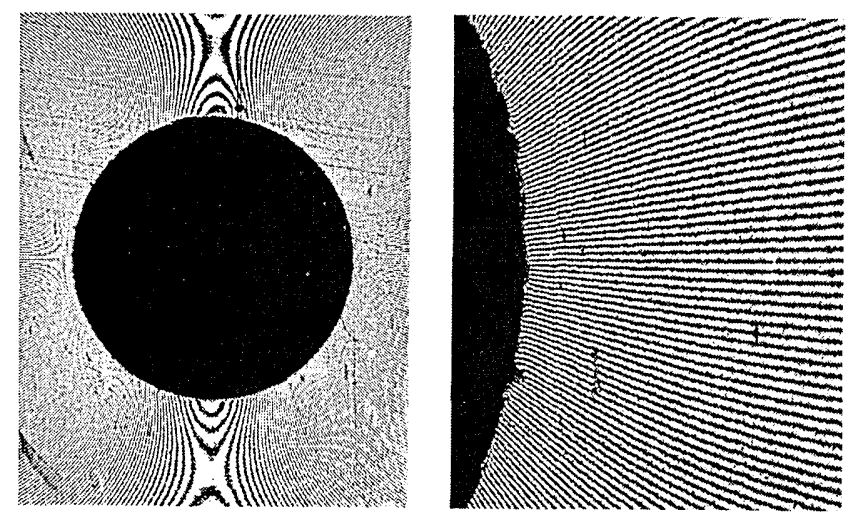

Fig. 3. Enlarged views of moire interferometry fringe pattern near the hole. $f=4000$ lines $/ \mathrm{mm}(101,600$ lines/in.) or $97.6 \%$ of the theoretical upper limit. Sensitivity is $0.25 \mu \mathrm{m} /$ fringe ( $9.8 \mu \mathrm{in}$./fringe). 
The fringes have excellent contrast or definition even where they are very closely spaced. The maximum fringe density at the hole is 24 fringes $/ \mathrm{mm}(610$ fringes/in.). Fringe orders in the field of view are extremely high.

Sensitivity to in-plane displacements corresponds to that of moire, with 4000 lines/mm $(101,600$ lines/in.) or a sensitivity of $0.25 \mu \mathrm{m} /$ fringe (9.8 $\mu \mathrm{in}$./fringes). If the application should warrant it, fringes can be interpolated to yield an order of magnitude finer resolution.

\section{v. Conclusions}

Moire interferometry with a reference grating frequency of 4000 lines/mm (101,600 lines/in.) was demonstrated. This corresponds to $97.6 \%$ of the theoretical upper limit of frequency and $97.6 \%$ of the theoretical upper limit of sensitivity.

Excellent fringe definition was obtained over the entire large field of view. The displacement-induced fringes were very closely packed and had very high information content. They prove that the range of effectiveness of moire interferometry extends close to the theoretical limit.

In moire interferometry fringe quality corresponds to that characteristic of two-beam interference with

Meetings Calendar continued from page 1614

1982

August

1-5 24th Rocky Mountain Conf., Denver W. Beard, USDA-ARS, P.O. Box E, Ft. Collins, Colo. 80522

16-18 Flow Visualization Techniques: Principles and Applications course, Ann Arbor W. Yang, U. Mich., Mech. Eng. \& App. Mech. Dept., 550 East Univ., Ann Arbor, Mich., 48109

21-27 SPIE 26th Ann. Int. Tech. Symp. \& Exhibit/15th Int. Cong. on High Speed Photography and Photonics, San Diego SPIE, P.O. Box 10, Bellingham, Wash. 98227

23-27 X-Ray \& Atomic Inner-Shell Physics Int. Conf., Eugene, Ore. B. Crasemann, Phys. Dept., U. Ore., Eugene, Ore. 97403

23-3 Sept. NATO Study Inst. Non-Linear Raman Spectroscopy and its Chemical Applications, Bad Windsheim W. Kiefer, Physikalisches Institut, Universitat Bayreuth, Postfach 3008, D-8580 Bayreuth, Federal Republic of Germany

\section{September}

? SPIE Industrial Applications of Infrared Thermography Conf., Milwaukee SPIE, P.O. Box 10, Bellingham, Wash. 98227

5-10 Precision and Speed in Close Range Photogrammetry Int Symp., York K. Atkinson, Dept. Photogrammetry \& Surveying, U. Coll. London, Gower St., London WC1E $6 B T$, England

6-11 VIIIth Int. Conf. on Raman Spectroscopy, Bordeaux J. Lascombe, VIIIth Int. Conf. on Raman Spect., Universite de Bordeaux I, 351, cours de la Liberation F33405 Talence, France coherent light. It is free of important limitations of a related method, high-sensitivity speckle interferometry, ${ }^{7}$ inasmuch as it offers extensive range (large displacements permitted), high and constant fringe contrast (independent of fringe order), and nonlocalization of fringes.

Support by the National Science Foundation and Engineering Science and Mechanics Department of Virginia Polytechnic Institute \& State University is greatly appreciated.

\section{References}

1. D. Post and W. A. Baracat, Exp. Mech. 21 (3), 100 (1981).

2. D. E. Bowles, D. Post, C. T. Herakovich, and D. R. Tenney, Proc. Soc. Exp. Stress Anal. 391 (1981); Exp. Mech. 21 (12), 441 (1981).

3. R. B. Watson and D. Post, Proc. Soc. Exp. Stress Anal. 414 (1981); Exp. Mech., to be published.

4. E. M. Weissman and D. Post, Proc. Soc. Exp. Stress Anal. 406, (1981); Exp. Mech., to be published.

5. D. Post, in Proceedings, Mechanics of Nondestructive Testing, W. W. Stinchcomb, Ed. (Plenum, New York, 1980).

6. M. L. Basehore, "Moire Interferometry for Out-of Plane Displacement Measurements," Ph.D. Dissertation, Virginia Polytechnic Institute \& State University, 1981.

7. V. J. Parks, Exp. Mech. 20 (6), 181 (1980).
8-10

$12-17$

19-23 4th Ann. Conf. Lasers in Graphics/Electronic Publishing in the 80's, Miami R. Dunn, 1131 Beaumont Circle, Vista, Calif. 92083

19-24 FACSS 9th Ann. Mtg., Phila. A. Zander, Spectrametrics, Inc., 204 Andover St., Andover, Mass. 01810

21-23 1st Int. Congr. on Applications of Lasers and ElectroOptics, Boston H. Lee, LIA Bus. Off., 5151 Monroe St., Toledo, Ohio 43623

21-24 VIII European Conf. on Optical Communication, Cannes Secretariat General ECOC 1982, 11 rue de Hameline, 75783 Paris Cedex 16, France

\section{October}

4-6

Technology for Space Astrophysics-The Next 30 Years Conf., Danbury D. McCarthy, Mail Station 879, Perkin-Elmer Corp., 100 Wooster Hgts. Rd., Danbury, Conn. 06810

4-8 Effective Engineering Management course, Boston Cont. Ed. Inst., 10889 Wilshire Blvd., Suite 1030, Los Angeles, Calif. 90024

4-8 IEEE's Industrial Applications Group, Electrostatics, Electrophotography and Electrostatic Precipitators, San Francisco M. Hirsh, 28 Spier Ave., Rochester, N.Y. 14620

5-8 Holographic Data Non-Destructive Testing Conf., Dubrovnik D. Vukicevic, IFS-Inst. Phys., U. Zagreb, YU/41000 Zagreb, Bijenicka 45, Dubrounik, Yugoslavia

continued on page 1633 